\title{
Quantum Einstein-Cartan theory with the Holst term
}

\author{
Ilya L. Shapiro ${ }^{1 *}$ and Poliane M. Teixeira ${ }^{\dagger}$ \\ Departamento de Física, ICE, Universidade Federal de Juiz de Fora \\ Campus Universitário - Juiz de Fora, MG, Brazil 36036-900
}

\begin{abstract}
Holst term represents an interesting addition to the EinsteinCartan theory of gravity with torsion. When this term is present the contact interactions between vector and axial vector fermion currents gain an extra parity-violating component. We re-derive this interaction using a simple representation for the Holst term. The same representation serves as a useful basis for the calculation of one-loop divergences in the theory with external fermionic currents and cosmological constant. Furthermore, we explore the possibilities of the on-shell version of renormalization group and construct the equations for the running of dimensionless parameters related to currents and for the effective Barbero-Immirzi parameter.
\end{abstract}

MSC: 81T15, 83D05 11.10.Gh, 04.50.Kd

PACS: $04.20 .-\mathrm{q}$ 98.80.-k

Keywords: Barbero-Immirzi parameter, Einstein-Cartan theory, Renormalization group.

\section{Introduction}

Einstein-Cartan theory attracts growing interest (see, e.g., [1], [2], [3] and references therein) because it represents a simplest possible extension of General Relativity (GR) related to the introduction of torsion field. The presence of torsion enables one to enrich the theory further by implementing the Holst term [4], which emerge naturally in the framework of loop quantum gravity $[5,6,1,7]$. This parity-violating term should attract a special interest since

*Also at Tomsk State Pedagogical University. Email address: shapiro@fisica.ufjf.br

$\dagger$ Email address: poliane@fisica.ufjf.br 
it can, in principle, yield some measurable observables for detecting quantum gravity. In order to better understand this point let us remember that in the Einstein-Cartan theory torsion becomes relevant only in the presence of fermion currents. After being integrated out, torsion provides contact interactions between such currents. Obviously, the main possibility for the Holst term, in this respect, is related to the generation of parity-violating contact interaction between vector and axial vector fermion currents. The first purpose of the present communication is to present a very simple derivation of the Holst term in terms of irreducible components of the torsion tensor. We show that the new term is the simplest possible parity-violating scalar, and hence the Barbero-Immirzi parameter [8, 9] can be seen as an extra non-minimal parity-violating extension of the Einstein-Cartan action. Using this new form we recalculate the contact interaction between fermion currents depending on Barbero-Immirzi parameter and meet good correspondence with the previous results of other authors $[10,6,1,11]$.

The main motivation for the Barbero-Immirzi parameter is related to Quantum Gravity (QG), so it is natural to see what can be the role of such term in the loop corrections. Since quantum GR and also quantum Einstein-Cartan theory is not renormalizable, this issue can not be addressed in the conventional framework of perturbative quantum field theory for the metric and torsion. The existing publications in this direction use very different approaches. The first of them is based on the functional renormalization group [12]. This powerful method is essentially non-perturbative, and in case of QG there is no perturbative limit, at least in the case of quantum GR. At the same time, there is a known difficulty related to the gauge-fixing dependence of the results of the functional renormalization group applied to gauge theories $[13,14]$ (see also many other references therein). In fact, the gauge-fixing dependence in this theory persists on-shell [14] and leads to the gauge dependent $S$-matrix and possibly all other relevant observables. One can expect that the same strong gauge dependence will take place also in the case of QG, and this creates certain difficulty for the physical interpretation of the results of this approach.

Another possibility is to rely on the renormalization group equations extracted from the quadratic one-loop divergences. This is technically possible, however the ambiguities which one usually meets in such a formulation are very strong and even go beyond the gauge fixing ambiguities. This aspect of QG has attracted significant interest recently, and the net result is that these ambiguities are generally uncontrollable (see, e.g., [15] and further references therein). For the Einstein-Cartan theory with the Barbero-Immirzi parameter this scheme was applied recently in [16], where the previous results for the one-loop divergences in the quantum GR with interacting fermion currents [17] have been used.

In the present work we use the third possibility for the quantum Einstein-Cartan theory. It is well-known that the pure quantum GR is renormalizable on-shell at the one-loop level 
[18]. This enables one to consider, for instance, the reduced on-shell version of renormalization group for the Newton constant and cosmological term [19]. Let us note that this approach can be extended to become more informative when the calculations are performed on the special background such as deSitter space [20], but our intention here is to follow a more simple method of [19]. A very nice feature of this approach is that the renormalization group equation for the dimensionless combination of the cosmological and Newton constants is gauge-fixing independent and, in this sense, is well defined. Of course, the on-shell renormalization group can not be seen as a completely consistent method, but it is a useful starting point to deal with the QG theory.

The on-shell one loop renormalization group has been generalized for the case of the Einstein-Cartan theory in [21], in the theory with an external axial vector current. It was shown that the theory remains on-shell renormalizable at first loop in the presence of such current and quantum torsion. Here we intend to generalize these considerations in two ways, namely by including an additional vector current and also by incorporating the Holst term. We shall analyze to which extent the on-shell renormalizability can be preserved in such a theory and also consider the on-shell renormalization group to the extent it is possible.

The paper is organized as follows. In Sect. 2, the classical consideration of the EinsteinCartan theory with the Holst term and two (vector and axial vector) currents is presented. The derivation of one-loop divergences and analysis of the on-shell renormalizability of the theory is described in Sect. 3. Sect. 4 contains the consideration of the on-shell renormalization group in the theory. Finally, in the last section we draw our conclusions and discuss possible perspectives for a future work.

\section{Simple representation for the Holst term}

In what follows we shall use the notations of $[22],{ }^{1}$ but will first reproduce the main formulas, for the convenience of the reader. The total action of gravity, including Einstein-Cartan and Holst terms has the form

$$
S_{E C}+S_{H}=-\frac{1}{\kappa^{2}} \int d^{4} x \sqrt{-g} \tilde{R}-\frac{1}{2 \gamma \kappa^{2}} \int d^{4} x \sqrt{-g} \varepsilon^{\alpha \beta \mu \nu} \tilde{R}_{\alpha \beta \mu \nu},
$$

where $G=\kappa^{2} / 16 \pi$ is Newton constant, also $16 \pi / \kappa^{2}=M_{P}^{2} . \quad \gamma$ is the Barbero-Immirzi parameter. The scalar curvature is $\tilde{R}=g^{\alpha \mu} g^{\beta \nu} \tilde{R}_{\alpha \beta \mu \nu}$ and $\tilde{R}_{\alpha \beta \mu \nu}$ is the curvature tensor depending on the metric $g_{\alpha \beta}$ and torsion $T_{\cdot \beta \gamma}^{\alpha}$. This curvature is defined on the basis of asymmetric connection

$$
\tilde{\Gamma}_{\beta \gamma}^{\alpha}-\tilde{\Gamma}_{\gamma \beta}^{\alpha}=T_{\cdot \beta \gamma}^{\alpha} \neq 0
$$

\footnotetext{
${ }^{1}$ One can use this reference and also many other sources, e.g., $[23,24,25]$ for the introduction to different aspects of gravity with torsion.
} 
Assuming that covariant derivative with torsion satisfies the metricity condition $\tilde{\nabla}_{\mu} g_{\alpha \beta}=0$, one can easily derive the relation between affine connection and Christoffel symbol $\Gamma_{\beta \gamma}^{\alpha}$,

$$
\tilde{\Gamma}_{\beta \gamma}^{\alpha}=\Gamma_{\beta \gamma}^{\alpha}+K_{\cdot \beta \gamma}^{\alpha}
$$

Here the contorsion tensor is

$$
K_{\cdot \beta \gamma}^{\alpha}=\frac{1}{2}\left(T_{\cdot \beta \gamma}^{\alpha}-T_{\beta \cdot \gamma}^{\alpha}-T_{\gamma \cdot \beta}^{\alpha}\right) .
$$

The corresponding relations for curvature tensor and scalar with torsion have the form

$$
\begin{aligned}
\tilde{R}_{\cdot \tau \alpha \beta}^{\lambda} & =R_{\cdot \tau \alpha \beta}^{\lambda}+\nabla_{\alpha} K_{\cdot \tau \beta}^{\lambda}-\nabla_{\beta} K_{\cdot \tau \alpha}^{\lambda}+K_{\cdot \gamma \alpha}^{\lambda} K_{\cdot \tau \beta}^{\gamma}-K_{\cdot \gamma \beta}^{\lambda} K_{\cdot \tau \alpha}^{\gamma} \\
\tilde{R} & =R+2 \nabla^{\lambda} K_{\cdot \lambda \tau}^{\tau}-K_{\tau \lambda}{ }^{\lambda} K_{\cdot \gamma \gamma}^{\tau \gamma}+K_{\tau \gamma \lambda} K^{\tau \lambda \gamma}
\end{aligned}
$$

where the quantities without tildes are Riemannian, without torsion.

One can introduce three irreducible components of torsion as follows:

$$
\begin{aligned}
\text { vector trace } & T_{\beta}=T_{\cdot \beta \alpha}^{\alpha}, \\
\text { axial vector trace } & S^{\nu}=\epsilon^{\alpha \beta \mu \nu} T_{\alpha \beta \mu}, \\
\text { tensor part } & q_{\cdot \beta \gamma}^{\alpha},
\end{aligned}
$$

when the last one satisfies the conditions $q_{\cdot \beta \alpha}^{\alpha}=0$ and $\epsilon^{\alpha \beta \mu \nu} q_{\alpha \beta \mu}=0$.

The generic torsion can be easily expressed as

$$
T_{\alpha \beta \mu}=\frac{1}{3}\left(T_{\beta} g_{\alpha \mu}-T_{\mu} g_{\alpha \beta}\right)-\frac{1}{6} \varepsilon_{\alpha \beta \mu \nu} S^{\nu}+q_{\alpha \beta \mu} .
$$

Now, replacing (10) into (4) and (6) we arrive at ${ }^{2}$

$$
\tilde{R}=R-2 \nabla_{\alpha} T^{\alpha}-\frac{2}{3} T_{\alpha} T^{\alpha}+\frac{1}{2} q_{\alpha \beta \gamma} q^{\alpha \beta \gamma}+\frac{1}{24} S_{\alpha} S^{\alpha}
$$

Finally, repeating the same operation with (5) and then with the integrand of the Holst term, we arrive at the relation, which was already reported in [10] (see also [28] for more detailed consideration and more complete list of references on the history of the parityviolating terms in Einstein-Cartan theory,

$$
\begin{aligned}
\varepsilon^{\alpha \beta \mu \nu} \tilde{R}_{\alpha \beta \mu \nu} & =-\nabla_{\mu} S^{\mu}-\frac{2}{3} S^{\mu} T_{\mu}+\frac{1}{2} \varepsilon^{\alpha \beta \mu \nu} q_{\cdot \alpha \beta}^{\lambda} q_{\lambda \mu \nu} \\
& =-\nabla_{\mu} S^{\mu}-\frac{2}{3} S \cdot T+\frac{1}{2} \varepsilon \cdot q \cdot q .
\end{aligned}
$$

In the last relation we have introduced condensed notations with dots for the contractions of two vectors and two tensors. The first of these notations will be used a lot in what follows.

\footnotetext{
${ }^{2}$ We correct a misprint in the coefficient of $T^{2}$ term in [22].
} 
One can see that the first part of the Holst term is rather simple in the representation (12). This term is nothing else but the simplest possible term violating parity. In fact, this term was not introduced as a non-minimal structure in the early works on quantum effects in gravity with torsion $[26,21]$ only because there was no interest to violate parity. For instance, the non-minimal structure $\varphi^{2} S^{\alpha} T_{\alpha}$ becomes relevant in the scalar sector if the parity-breaking nonminimal terms $\bar{\psi} \gamma^{\alpha} S_{\alpha} \psi$ or $\bar{\psi} \gamma^{\alpha} \gamma^{5} T_{\alpha} \psi$ are introduced. In this case the Holst term can be easily obtained as part of the induced action (extended Einstein-Cartan) of gravity with torsion, e.g., it can result from some phase transition scheme, including spontaneous symmetry breaking.

In order to better understand the effect of the Holst term, let us include vector $V^{\mu}$ and axial vector $A^{\mu}$ fermion currents,

$$
V^{\mu}=\eta_{2}\left\langle\bar{\psi} \gamma^{\mu} \psi\right\rangle \text { and } \quad A^{\mu}=\eta_{1}\left\langle\bar{\psi} \gamma^{\mu} \gamma^{5} \psi\right\rangle
$$

Let us note that the presence of non-minimal parameters $\eta_{1,2}$ is the condition of consistency of the theory at the quantum level, especially if scalar fields and Yukawa interactions of these fields with fermions are present [26] (see also [27] and [22] for extended discussions of this issue). For the sake of compactness of notations in the quantum part of the work, it is better to introduce also rescaled currents $J^{\mu}=-\kappa^{2} A^{\mu}$ and $W^{\mu}=-\kappa^{2} V^{\mu}$, such that the total action becomes

$$
\begin{aligned}
S_{t} & =S_{E C}+S_{H}+\int d^{4} x \sqrt{-g}(V \cdot T+A \cdot S) \\
& =-\frac{1}{\kappa^{2}} \int d^{4} x \sqrt{-g}\left\{R+2 \Lambda-\frac{2}{3} T^{2}+\frac{1}{2} q^{2}+\frac{1}{24} S^{2}\right. \\
& \left.-\frac{1}{3 \gamma} S \cdot T-\frac{1}{2 \gamma} \nabla_{\mu} S^{\mu}+\frac{1}{4 \gamma} \varepsilon \cdot q \cdot q+S \cdot J+T \cdot W\right\},
\end{aligned}
$$

where we also used compact notations $S^{2}=S_{\mu} S^{\mu}, T^{2}=T_{\mu} T^{\mu}$ and $q^{2}=q_{\mu \nu \tau} q^{\mu \nu \tau}$.

As usual in the Einstein-Cartan theory, torsion is not dynamical field and can be integrated out. The dynamical equations for different components of torsion have the form

$$
\begin{aligned}
-\frac{4}{3} T^{\alpha}-\frac{1}{3 \gamma} S^{\alpha}+W^{\alpha} & =0 \\
\frac{1}{12} S^{\alpha}-\frac{1}{3 \gamma} T^{\alpha}+J^{\alpha} & =0, \\
q^{\alpha \beta \gamma} & =0 .
\end{aligned}
$$

According to the last equation we will not consider the component $q^{\alpha \beta \gamma}$ further. The first two equations can be easily solved in the form

$$
\begin{aligned}
T^{\alpha} & =\frac{3 \gamma}{1+\gamma^{2}}\left(J^{\alpha}+\frac{\gamma}{4} W^{\alpha}\right), \\
S^{\alpha} & =\frac{3 \gamma}{1+\gamma^{2}}\left(W^{\alpha}-4 \gamma J^{\alpha}\right) .
\end{aligned}
$$


One can observe that the presence of parity-violating parameter $\gamma$ leads to the mixing between vector and axial vector currents. In principle, this mixing may have some strong phenomenological consequences, and it would be interesting to explore its consequences in particle physics. Such investigation could lead to the upper bounds of certain combinations of the Barbero-Immirzi parameter $\gamma$ and the non-minimal parameters $\eta_{1,2}$, introduced in (13). However, in the present work our purpose is not phenomenology, instead we shall focus our attention on more formal aspects of the theory, related to QG.

The dynamical equation for the metric in the theory (14) leads to the on-shell relations

$$
R_{\mu \nu}=D_{\mu \nu}-g_{\mu \nu}\left(\Lambda+\frac{1}{2} S \cdot J+\frac{1}{2} T \cdot W\right)
$$

where we introduced a useful notation

$$
D_{\mu \nu}=\frac{2}{3} T_{\mu} T_{\nu}-\frac{1}{24} S_{\mu} S_{\nu}+\frac{1}{6 \gamma}\left(S_{\mu} T_{\nu}+S_{\nu} T_{\mu}\right) \quad \text { and also } \quad D=D_{\mu}^{\mu} .
$$

Finally, replacing (16) into (17), after some algebra we arrive at the on-shell relations

$$
\begin{aligned}
\left.R_{\mu \nu}\right|_{\text {on-shell }} & =-\Lambda g_{\mu \nu}+\frac{3 \gamma}{1+\gamma^{2}}\left(2 \gamma J^{2}-\frac{\gamma}{8} W^{2}-J \cdot W\right) g_{\mu \nu} \\
& +\frac{3 \gamma}{1+\gamma^{2}}\left[\frac{\gamma}{8} W_{\mu} W_{\nu}-2 \gamma J_{\mu} J_{\nu}+\frac{1}{2}\left(W_{\mu} J_{\nu}+W_{\nu} J_{\mu}\right)\right]
\end{aligned}
$$

and

$$
\left.R\right|_{\text {on-shell }}=-4 \Lambda+\frac{3 \gamma}{1+\gamma^{2}}\left[6 \gamma J^{2}-\frac{3 \gamma}{8} W^{2}-3 W \cdot J\right]
$$

Finally, for the total action (14) on-shell we obtain

$$
\left.S_{t}\right|_{o n-s h e l l}=-\frac{1}{\kappa^{2}} \int d^{4} x \sqrt{-g}\left\{\frac{3 \gamma}{1+\gamma^{2}}\left(4 \gamma J^{2}-2 J \cdot W-\frac{\gamma}{4} W^{2}\right)-2 \Lambda\right\}
$$

A simple observation concerning this action is as follows. In the limit $\gamma \rightarrow \infty$ the mixed term with $(J \cdot W)$ goes to zero. This is of course a natural feature, because this parityviolating term is only due to the presence of the Holst term. This detail is an illustration of the possible effects of the Holst term on the interaction between the two vector currents.

\section{One-loop divergences off- and on-shell}

The divergences must be calculated on the basis of the off-shell action (14). We shall treat $g_{\mu \nu}, S_{\alpha}$ and $T_{\alpha}$ as quantum fields while $W^{\alpha}$ and $J^{\alpha}$ will be taken as external sources. The Gaussian path integrals over $S_{\alpha}$ and $T_{\alpha}$ do not generate divergences, because the 
corresponding bilinear forms are c-number operators. This means that integrations over these variables is greatly simplified. Let us see this in more details.

Consider the background field method for the action (14) and shift the field variable into background and quantum parts according to

$$
g_{\mu \nu} \rightarrow g_{\mu \nu}^{\prime}=g_{\mu \nu}+\kappa h_{\mu \nu}, \quad S_{\mu} \rightarrow S_{\mu}^{\prime}=S_{\mu}+\kappa \sigma_{\mu}, \quad T_{\mu} \rightarrow T_{\mu}^{\prime}=T_{\mu}+\kappa t_{\mu} .
$$

The one-loop effective action depends on the bilinear in respect to the quantum fields $h_{\mu \nu}, \sigma_{\mu}, t_{\mu}$ part of the action. Since we are going to work with on-shell quantities, the choice of the gauge fixing is irrelevant. For the sake of simplicity we consider

$$
S_{g f}=-\frac{1}{2 \theta} \int d^{4} x \sqrt{-g} \chi_{\mu} \chi^{\mu}, \quad \text { where } \quad \chi_{\mu}=\nabla_{\lambda} h_{\mu}^{\lambda}-\frac{\omega}{2} \nabla_{\mu} h
$$

and chose the gauge fixing parameters in a way that leads to the minimal bilinear form of the action, namely $\theta=\omega=1$.

The expansion performs as usual (see, e.g., [27] for details) and after some algebra we arrive at

$$
\begin{aligned}
S_{t}^{(2)}+S_{g f} & =-\int d^{4} x \sqrt{-g}\left\{h ^ { \mu \nu } \left[\frac{1}{4}\left(\delta_{\mu \nu, \alpha \beta}-\frac{1}{2} g_{\mu \nu} g_{\alpha \beta}\right) \square+\frac{1}{2} R_{\mu \alpha \nu \beta}\right.\right. \\
& +\frac{1}{2} g_{\nu \beta} R_{\mu \alpha}-\frac{1}{4}\left(g_{\mu \nu} R_{\alpha \beta}+g_{\alpha \beta} R_{\mu \nu}\right) \\
& -\frac{1}{4}\left(\delta_{\mu \nu, \alpha \beta}-\frac{1}{2} g_{\mu \nu} g_{\alpha \beta}\right)\left(R+2 \Lambda-\frac{2}{3} T^{2}+\frac{1}{24} S^{2}-\frac{1}{3 \gamma} S \cdot T+S \cdot J+T \cdot W\right) \\
& -\frac{1}{96}\left(g_{\mu \nu} S_{\alpha} S_{\beta}+g_{\alpha \beta} S_{\mu} S_{\nu}\right)-\frac{2}{3} g_{\mu \alpha} T_{\nu} T_{\beta}+\frac{1}{6}\left(g_{\mu \nu} T_{\alpha} T_{\beta}+g_{\alpha \beta} T_{\mu} T_{\nu}\right) \\
& \left.-\frac{1}{6 \gamma} g_{\mu \alpha}\left(S_{\nu} T_{\beta}+S_{\beta} T_{\nu}\right)+\frac{1}{12 \gamma}\left(g_{\mu \nu} S_{\alpha} T_{\beta}+g_{\alpha \beta} S_{\mu} T_{\nu}\right)+\frac{1}{24} g_{\mu \alpha} S_{\nu} S_{\beta}\right] h^{\alpha \beta} \\
& +\frac{1}{24} g^{\mu \nu} \sigma_{\mu} \sigma_{\nu}-\frac{2}{3} g^{\mu \nu} t_{\mu} t_{\nu}-\frac{1}{6 \gamma}\left(\sigma_{\mu} g^{\mu \nu} t_{\nu}+\sigma_{\nu} g^{\mu \nu} t_{\mu}\right) \\
& +h^{\mu \nu}\left[-\frac{1}{12} S_{\mu} \sigma_{\nu}+\frac{4}{3} T_{\mu} t_{\nu}+\frac{1}{3 \gamma}\left(S_{\mu} t_{\nu}+T_{\mu} \sigma_{\nu}\right)\right]+\left[\frac{1}{24} g_{\alpha \beta} S_{\mu} \sigma^{\mu}\right. \\
& \left.\left.-\frac{2}{3} g_{\alpha \beta} T_{\mu} t^{\mu}-\frac{1}{6 \gamma} g_{\alpha \beta}\left(T^{\mu} \sigma_{\mu}+S^{\mu} t_{\mu}\right)+\frac{1}{2} g_{\alpha \beta} \sigma_{\mu} J^{\mu}+\frac{1}{2} g_{\alpha \beta} t_{\mu} W^{\mu}\right] h^{\alpha \beta}\right\},
\end{aligned}
$$

where $\delta_{\mu \nu, \alpha \beta}=(1 / 2)\left(g_{\mu \alpha} g_{\nu \beta}+g_{\mu \beta} g_{\nu \alpha}\right)$. A relevant observation is that the path integral over $\sigma_{\nu}$ and $t_{\mu}$ has the form

$$
I=\int d t_{\mu} d \sigma_{\nu} \exp \left\{i\left[\frac{1}{2}\left(\sigma_{\mu} t_{\mu}\right)\left(K^{\mu \nu}\right)\left(\begin{array}{c}
\sigma_{\nu} \\
t_{\nu}
\end{array}\right)+\left(\sigma_{\mu} t_{\mu}\right)\left(\begin{array}{l}
a^{\mu} \\
b^{\mu}
\end{array}\right)\right]\right\}
$$

where $K^{\mu \nu}$ is a $c$-matrix and $a^{\mu}, b^{\mu}$ form a column depending on the background fields. This non-derivative Gaussian integration gives

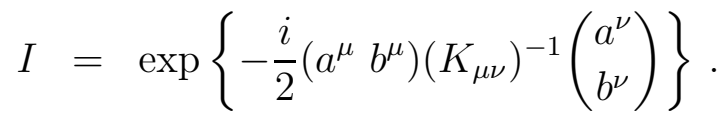


This is the same result which one could obtain just using the classical equations of motion for the two components of torsion $\sigma_{\nu}$ and $t_{\mu}$. Since our intention is to calculate the on-shell effective action, it means that we can simply ignore path integrals over $\sigma_{\nu}$ and $t_{\mu}$. That means there is no need to perform the shift of $S_{\nu}$ and $T_{\mu}$ in (22), instead one can directly use corresponding classical equations of motion in the result of the integration over quantum metric $h_{\mu \nu}$.

Finally, the relevant part of the bilinear expansion is

$$
\begin{aligned}
S_{t}^{(2)}+S_{g f} & =-\int d^{4} x \sqrt{-g} h^{\mu \nu}\left\{\frac{1}{4}\left(\delta_{\mu \nu, \alpha \beta}-\frac{1}{2} g_{\mu \nu} g_{\alpha \beta}\right) \square+\frac{1}{2} R_{\mu \alpha \nu \beta}+\frac{1}{2} g_{\nu \beta} R_{\mu \alpha}\right. \\
& \left.-\frac{1}{4}\left(g_{\mu \nu} R_{\alpha \beta}+g_{\alpha \beta} R_{\mu \nu}\right)-\frac{1}{4}\left(\delta_{\mu \nu, \alpha \beta}-\frac{1}{2} g_{\mu \nu} g_{\alpha \beta}\right) X+\frac{1}{4} Y_{\mu \nu, \alpha \beta}\right\} h^{\alpha \beta}
\end{aligned}
$$

where

$$
X=R+2 \Lambda-\frac{2}{3} T^{2}+\frac{1}{24} S^{2}-\frac{1}{3 \gamma} S \cdot T+S \cdot J+T \cdot W
$$

and

$$
\begin{aligned}
Y_{\mu \nu, \alpha \beta} & =\frac{1}{6} g_{\mu \alpha} S_{\nu} S_{\beta}-\frac{1}{24}\left(g_{\mu \nu} S_{\alpha} S_{\beta}+g_{\alpha \beta} S_{\mu} S_{\nu}\right)-\frac{8}{3} g_{\mu \alpha} T_{\nu} T_{\beta}+\frac{2}{3}\left(g_{\mu \nu} T_{\alpha} T_{\beta}+g_{\alpha \beta} T_{\mu} T_{\nu}\right) \\
& -\frac{2}{3 \gamma} g_{\mu \alpha}\left(S_{\nu} T_{\beta}+S_{\beta} T_{\nu}\right)+\frac{1}{3 \gamma}\left(g_{\mu \nu} S_{\alpha} T_{\beta}+g_{\alpha \beta} S_{\mu} T_{\nu}\right)
\end{aligned}
$$

Furthermore, the equation (27) can be rewritten as

$$
\begin{aligned}
S_{t}^{(2)}+S_{g f} & =-\int d^{4} x \sqrt{-g} h^{\mu \nu}\left(\frac{1}{4} K_{\mu \nu, \alpha \beta} \square+\frac{1}{4} M_{\mu \nu, \alpha \beta}\right) h^{\alpha \beta} \\
& =-\frac{1}{4} \int d^{4} x \sqrt{-g} h^{\mu \nu} K_{\mu \nu, \alpha \beta}\left[\delta_{\rho \sigma}^{\alpha \beta,} \square+\hat{\Pi}_{\rho \sigma}^{\alpha \beta}\right] h^{\rho \sigma},
\end{aligned}
$$

where

$$
\begin{aligned}
\hat{\Pi}_{\alpha \beta, \rho \sigma} & =2 R_{\rho \alpha \sigma \beta}+2 g_{\sigma \beta} R_{\rho \alpha}-\left(g_{\rho \sigma} R_{\alpha \beta}+g_{\alpha \beta} R_{\rho \sigma}\right) \\
& +\frac{1}{2} g_{\rho \sigma} g_{\alpha \beta} R-\delta_{\rho \sigma, \alpha \beta} X+Y_{\rho \sigma, \alpha \beta}-\frac{1}{2} g_{\rho \sigma} Y_{\rho \sigma, \alpha \beta} g^{\mu \nu}
\end{aligned}
$$

and

$$
K_{\mu \nu, \alpha \beta}^{-1}=K_{\mu \nu, \alpha \beta}=\delta_{\mu \nu, \alpha \beta}-\frac{1}{2} g_{\mu \nu} g_{\alpha \beta} .
$$

The one-loop contribution is given by the standard expression

$$
\Gamma^{(1)}=\frac{i}{2} \operatorname{Tr} \ln \{\hat{K} \cdot(\hat{\square}+\hat{\Pi})\}-i \operatorname{Tr} \ln \hat{H}_{\text {ghost }} .
$$

The ghost part does not depend on torsion or external currents, hence the corresponding contribution will be identical to the standard one for Einstein gravity [18]. Let us, therefore, 
concentrate on the first term in (33). As far as $\operatorname{Tr} \ln \hat{K}=\operatorname{Tr} \ln \hat{K}_{\mu \nu, \alpha \beta}$ does not contribute to the divergences, they depend only on the matrix $\hat{\Pi}_{\rho \sigma}^{\alpha \beta}$ and also on the contribution of the Faddeev-Popov ghosts.

The practical calculation of divergences follows the standard scheme [18] and we will avoid boring the reader with the details. The result for the divergent part of the one-loop effective action can be conveniently expressed via the tensor quantity (18) and has the following final form:

$$
\begin{aligned}
\bar{\Gamma}_{\text {div }}^{(1)} & =-\frac{1}{\varepsilon} \int d^{4} x \sqrt{-g}\left\{\frac{53}{45} E+\frac{7}{10} R_{\mu \nu}^{2}+\frac{1}{60} R^{2}+8 D_{\mu \nu} D^{\mu \nu}-2 D^{2}\right. \\
& \left.+\frac{26}{3} R\left(\Lambda+\frac{1}{2} S \cdot J+\frac{1}{2} T \cdot W\right)+20\left(\Lambda+\frac{1}{2} S \cdot J+\frac{1}{2} T \cdot W\right)^{2}\right\},
\end{aligned}
$$

where $E=R_{\mu \nu \alpha \beta}^{2}-4 R_{\mu \nu}^{2}+R^{2}$ is the Lagrangian density of the Gauss-Bonnet term (Euler density). Finally, $\varepsilon=(4 \pi)^{2}(n-4)$ is the parameter of dimensional regularization. Let us remark that (34) is relatively simple due to some unexpected cancellations, for example of the $D R, D_{\mu \nu} R^{\mu \nu}$ and a few other possible structures.

In order to formulate the on-shell renormalization group, we need to use the classical equations of motion (16), (18), (19) and (20) in eq. (34). After some algebra we arrive at the result

$$
\begin{aligned}
\left.\bar{\Gamma}_{\text {div }}^{(1)}\right|_{\text {on-shell }} & =-\frac{1}{\varepsilon} \int d^{4} x \sqrt{-g}\left\{\frac{53}{45} E-\frac{58}{5} \Lambda^{2}+\frac{81 \gamma^{4} J^{4}}{\left(1+\gamma^{2}\right)^{2}}+\frac{81}{256} \frac{\gamma^{4} W^{4}}{\left(1+\gamma^{2}\right)^{2}}\right. \\
& +\frac{27}{40} \frac{\gamma^{2}\left(43 \gamma^{2}+58\right)}{\left(1+\gamma^{2}\right)^{2}} W^{2} \cdot J^{2}+\frac{241 \gamma}{40\left(1+\gamma^{2}\right)}\left(16 \gamma J^{2}-\gamma W^{2}-8 W \cdot J\right) \Lambda \\
& \left.-\frac{27 \gamma^{2}(W \cdot J)}{80\left(1+\gamma^{2}\right)^{2}}\left[\left(56+116 \gamma^{2}\right)(W \cdot J)+240 \gamma J^{2}-15 \gamma W^{2}\right]\right\} .
\end{aligned}
$$

An important difference between the expressions (34) and (35) is related to the gauge fixing dependence. The effective action (34) has a lot of ambiguity related to the choice of the parameters $\theta, \omega$ in the action (23). In fact, significant part of the terms can be modified or even eliminated by an appropriate choice of these parameters [29]. On the other hand, there is no such gauge dependence in the one-loop divergences for the on-shell effective action [19] (see also more detailed consideration in [30]), so the coefficients in the action (35) do not suffer from this ambiguity.

\section{On-shell renormalization group}

Our purpose is to construct the reduced on-shell version of the Minimal Subtraction renormalization group. We shall use dimensional regularization and hence it is necessary to 
formulate both classical on-shell action (21) and the on-shell counterterm in $n$ space-time dimensions. The corresponding expressions can be written in terms of the new notations

$$
\tilde{\lambda}=\alpha_{1} \lambda_{1}+\alpha_{2} \lambda_{2}+\alpha_{3} \lambda_{3}+\alpha_{4} \lambda_{4}
$$

where

$$
\lambda_{1}=\kappa^{2} \Lambda, \quad \lambda_{2}=\kappa^{2} J^{2}, \quad \lambda_{3}=\kappa^{2} W^{2}, \quad \lambda_{4}=\gamma \kappa^{2}(W \cdot J)
$$

on one side and

$$
\begin{aligned}
\widetilde{\sigma} & =\Omega_{11} \lambda_{1}^{2}+\Omega_{22} \lambda_{2}^{2}+\Omega_{33} \lambda_{3}^{2}+\Omega_{44} \lambda_{4}^{2} \\
& +\Omega_{12} \lambda_{1} \lambda_{2}+\Omega_{13} \lambda_{1} \lambda_{3}+\Omega_{14} \lambda_{1} \lambda_{4}+\Omega_{23} \lambda_{2} \lambda_{3}+\Omega_{24} \lambda_{2} \lambda_{4}+\Omega_{34} \lambda_{3} \lambda_{4}
\end{aligned}
$$

on another side.

The classical action and one-loop counterterms, both on-shell (classical) have the form

$$
\begin{aligned}
\left.S_{t}\right|_{\text {on-shell }} & =-\frac{1}{\kappa^{4}} \int d^{n} x \sqrt{-g} \mu^{n-4} \widetilde{\lambda}, \\
\left.\Delta S^{(1)}\right|_{\text {on-shell }} & =\frac{1}{\varepsilon} \cdot \frac{1}{\kappa^{4}} \int d^{n} x \sqrt{-g} \mu^{n-4} \widetilde{\sigma} .
\end{aligned}
$$

The coefficients in the expressions (36) and (38) can be taken directly from eqs. (21) and (35)

$$
\alpha_{1}=-2, \quad \alpha_{2}=\frac{12 \gamma^{2}}{\left(1+\gamma^{2}\right)}, \quad \alpha_{3}=-\frac{3 \gamma^{2}}{4\left(1+\gamma^{2}\right)}, \quad \alpha_{4}=-\frac{6}{\left(1+\gamma^{2}\right)}
$$

and

$$
\begin{aligned}
& \Omega_{11}=-\frac{58}{5}, \quad \Omega_{12}=\frac{482}{5} \frac{\gamma^{2}}{\left(1+\gamma^{2}\right)}, \quad \Omega_{13}=-\frac{241}{40} \frac{\gamma^{2}}{\left(1+\gamma^{2}\right)}, \quad \Omega_{14}=-\frac{241}{5\left(1+\gamma^{2}\right)}, \\
& \Omega_{22}=\frac{81 \gamma^{4}}{\left(1+\gamma^{2}\right)^{2}}, \quad \Omega_{23}=\frac{27}{40} \frac{\gamma^{2}\left(43 \gamma^{2}+58\right)}{\left(1+\gamma^{2}\right)^{2}}, \quad \Omega_{24}=-\frac{81 \gamma^{2}}{\left(1+\gamma^{2}\right)^{2}}, \\
& \Omega_{33}=\frac{81}{256} \frac{\gamma^{4}}{\left(1+\gamma^{2}\right)^{2}}, \quad \Omega_{34}=\frac{81}{16} \frac{\gamma^{2}}{\left(1+\gamma^{2}\right)^{2}}, \quad \Omega_{44}=-\frac{\left(378+783 \gamma^{2}\right)}{20} \frac{1}{\left(1+\gamma^{2}\right)^{2}} .
\end{aligned}
$$

Let us note that consistent formulation of renormalization group for both cosmological constant and Newton constant (related to the inverse $\kappa$ of the re-scaled Planck mass) is definitely impossible since we are working in the framework of the on-shell renormalization group. The form of the classical action (39) and the counterterms (40) indicate that there is no possibility to study renormalization of $\kappa$ in this framework, so in what follows we will pursue only the aim of constructing the renormalization group equations for effective 
charges $\lambda_{1}, \lambda_{2}, \lambda_{3}$ and $\lambda_{4}$, defined in (37). One can also see this method as working in the Planck units, where all quantities become dimensionless.

The on-shell renormalized action has the form which follows from eqs. (39) and (40). Then the on-shell one-loop divergences can be removed by means of renormalization transformation

$$
\widetilde{\lambda}_{0}=\mu^{(n-4)}\left(\widetilde{\lambda}-\frac{\widetilde{\sigma}}{\varepsilon}\right)
$$

As far as $\widetilde{\lambda}_{0}$ does not depend on $\mu$, the last relation implies that

$$
(n-4)\left(\widetilde{\lambda}-\frac{\widetilde{\sigma}}{\varepsilon}\right)+\left(\mu \frac{d \widetilde{\lambda}}{d \mu}-\frac{\mu}{\varepsilon} \frac{d \widetilde{\sigma}}{d \mu}\right)=0 .
$$

Assuming that the divergent terms cancel, and using the homogeneity property of $\tilde{\sigma}$, we arrive at the general $\beta$-function for $\tilde{\lambda}$ in $n$ space-time dimensions,

$$
\beta_{\tilde{\lambda}}^{n}=-(n-4) \tilde{\lambda}-\frac{\widetilde{\sigma}}{(4 \pi)^{2}} .
$$

Since our intention to to explore the renormalization group in $n=4$, we have to take the limit $n \rightarrow 4$, to arrive at the general renormalization group equation

$$
\frac{d \widetilde{\lambda}}{d t}=\mu \frac{d \widetilde{\lambda}}{d \mu}=\beta_{\widetilde{\lambda}}=-\frac{\widetilde{\sigma}}{(4 \pi)^{2}},
$$

where we introduced a useful parameter $t=\ln \left(\mu / \mu_{0}\right)$.

The next part of the work will be to extract the equations for individual effective charges $\lambda_{1}, \lambda_{2}, \lambda_{3}$ and $\lambda_{4}$ from the single equation (46). This situation is definitely more complicated than the one in the usual renormalizable theories, and represents a necessary element of the more tricky scheme of the on-shell renormalization group.

The case of the parameter $\lambda_{1}$ has been considered in the paper [19], where the on-shell renormalization group was invented. Let us suppose that the renormalization group equation for the cosmological constant $\lambda_{1}$ does not depend on the presence of external currents $J^{\mu}$ and $W^{\mu}$. Setting $J^{\mu}=W^{\mu}=0$ we get $\lambda_{2,3,4}=0$ and then the eq. (46) transforms into

$$
\alpha_{1} \frac{d \lambda_{1}}{d t}=-\frac{1}{(4 \pi)^{2}} \Omega_{11} \lambda_{1}^{2} .
$$

Taking $\alpha_{1}$ and $\Omega_{11}$ from (41) and (42), one can immediately obtain the corresponding equation of [19],

$$
\frac{d \lambda_{1}}{d t}=-\frac{29}{5(4 \pi)^{2}} \lambda_{1}^{2}
$$


indicating an asymptotic freedom for the dimensionless cosmological constant in the UV for a positive cosmological constant and in the IR for a negative cosmological constant.

One can follow similar approach for another effective charge, $\lambda_{2}$. In this case one has to assume that when we set $\Lambda=0$ and $W^{\mu}=0$, the on-shell renormalization group equation for the effective charge related only to $J^{\mu}$ does not change. Then the considerations similar to the ones which led us to (47) and (48) provide us with the equation

$$
\frac{d \lambda_{2}}{d t}=\beta_{2}=-\frac{\Omega_{22}}{\alpha_{2}(4 \pi)^{2}} \lambda_{2}^{2}=-b_{2}^{2} \lambda_{2}^{2}=-\frac{27}{4(4 \pi)^{2}} \frac{\gamma^{2}}{\left(1+\gamma^{2}\right)} \lambda_{2}^{2},
$$

indicating an asymptotic freedom for the dimensionless quantity $\lambda_{2}$ in the UV, in case the vector $J^{\mu}$ is time-like, and in the IR in case the same vector is space-like.

In a similar way one can obtain the equation for the third parameter

$$
\frac{d \lambda_{3}}{d t}=\beta_{3}=-\frac{\Omega_{33}}{\alpha_{3}(4 \pi)^{2}} \lambda_{3}^{2}=b_{3}^{2} \lambda_{3}^{2}=\frac{27}{64(4 \pi)^{2}} \frac{\gamma^{2}}{\left(1+\gamma^{2}\right)} \lambda_{3}^{2} .
$$

In this case we observe the asymptotic freedom for the dimensionless quantity $\lambda_{3}$ in the UV if vector $W^{\mu}$ is space-like, and in the IR in case this vector is time-like. For the sake of simplicity, we shall assume that the initial value of $\lambda_{2}\left(\mu_{0}\right)=\lambda_{2}^{0}$ is positive and that the initial value $\lambda_{3}\left(\mu_{0}\right)=\lambda_{3}^{0}$ is negative. In this case we have asymptotic freedom for both charges in UV and will try to explore this limit in what follows. It is important to note that the signs of $\lambda_{2}$ or $\lambda_{3}$ are not limited by the arguments of stability or alike, in particular because they correspond to the properties of external (non-dynamical) currents.

Now we can start solving a more complicated problem of formulating the on-shell renormalization group equation for the effective charge $\lambda_{4}(t)$ and eventually for the effective Barbero-Immirzi parameter $\gamma(t)$. By subtracting eqs. (49) and (50) with the factors $\alpha_{2}$ and $\alpha_{3}$, from eq. (46) we obtain

$$
\alpha_{4} \frac{d \lambda_{4}}{d t}=\frac{1}{(4 \pi)^{2}}\left(-\widetilde{\sigma}+\Omega_{22} \lambda_{2}^{2}+\Omega_{33} \lambda_{3}^{2}\right)
$$

that directly brings us to

$$
(4 \pi)^{2} \frac{d \lambda_{4}}{d t}=-\frac{1}{\alpha_{4}}\left(\Omega_{44} \lambda_{4}^{2}+\Omega_{23} \lambda_{2} \lambda_{3}+\Omega_{24} \lambda_{2} \lambda_{4}+\Omega_{34} \lambda_{3} \lambda_{4}\right) .
$$

This is the renormalization group equation for the running parameter $\lambda_{4}(t)$. The $\beta$-function here depends on $\lambda_{2}(t)$ and $\lambda_{3}(t)$, so the first impression is that one can solve eq. (51) only after solving eqs. (49) and (50). However, the real situation is much more complicated. The parameter $\lambda_{4}$ is strongly related to $\lambda_{2}$ and $\lambda_{3}$, because all three constants are constructed from two fermion currents, $J^{\alpha}$ and $W^{\alpha}$, via eq. (37). In fact, we have found the room for an independent equation (51) only because $\lambda_{4}$ depends not just on the magnitude of 
the currents $J^{\alpha}$ and $W^{\alpha}$, but also on the angle between them and on the Barbero-Immirzi parameter $\gamma$. In what follows we assume that the mentioned angle does not run with the scale. This feature enables one to construct the renormalization group equation for $\gamma$.

Let us derive the renormalization group equation for the Barbero-Immirzi parameter. For this end we return to the equations (49) and (50). Since $\kappa$ is a universal constant (inverse Planck mass), one has to assume that the external currents themselves are running quantities, that means $J^{\alpha}=J^{\alpha}(\mu)$ and $W^{\alpha}=W^{\alpha}(\mu)$. By using (37), one can rewrite (49) and $(50)$ as

$$
\begin{aligned}
& \frac{d \lambda_{2}}{d t}=\kappa^{2} \frac{d J^{2}}{d t}=2 \kappa^{2} J^{\alpha} \frac{d J_{\alpha}}{d t}=-\frac{\Omega_{22} \lambda_{2}^{2}}{\alpha_{2}(4 \pi)^{2}}=\beta_{2} \\
& \frac{d \lambda_{3}}{d t}=\kappa^{2} \frac{d W^{2}}{d t}=2 \kappa^{2} W^{\alpha} \frac{d W_{\alpha}}{d t}=-\frac{\Omega_{33} \lambda_{3}^{2}}{\alpha_{3}(4 \pi)^{2}}=\beta_{3} .
\end{aligned}
$$

Let us make a natural assumption that

$$
\frac{d J_{\alpha}}{d t}=\Theta_{2} J_{\alpha}
$$

Than it is easy to show that the

$$
\Theta_{2}=-\frac{\Omega_{22} \kappa^{2} J^{2}}{2 \alpha_{2}(4 \pi)^{2}}
$$

In the same way, we find

$$
\frac{d W_{\alpha}}{d t}=\Theta_{3} W_{\alpha}, \quad \text { where } \quad \Theta_{3}=-\frac{\Omega_{33} \kappa^{2} W^{2}}{2 \alpha_{3}(4 \pi)^{2}} .
$$

Then we have two relations,

$$
\frac{d J_{\alpha}}{d t}=-\frac{\Omega_{22} \kappa^{2} J^{2}}{2 \alpha_{2}(4 \pi)^{2}} J_{\alpha} \quad \text { and } \quad \frac{d W_{\alpha}}{d t}=-\frac{\Omega_{33} \kappa^{2} W^{2}}{2 \alpha_{3}(4 \pi)^{2}} W_{\alpha}
$$

As far as $\lambda_{4}=\gamma \kappa^{2}(W \cdot J)$, the renormalization group equation for $\lambda_{4}$ is a consequence of equations (56) and the running of $\gamma$, which we also want to find. In this way one can obtain

$$
\frac{d \lambda_{4}}{d t}=\kappa^{2}\left(\frac{d \gamma}{d t} W \cdot J+\gamma J^{\alpha} \frac{d W_{\alpha}}{d t}+\gamma W^{\alpha} \frac{d J_{\alpha}}{d t}\right)
$$

Replacing (51) into (57) and using eqs. (56), we arrive at

$$
(4 \pi)^{2} \frac{1}{\gamma} \frac{d \gamma}{d t}=-\frac{\Omega_{44} \lambda_{4}}{\alpha_{4}}+\lambda_{2}\left(\frac{\Omega_{22}}{2 \alpha_{2}}-\frac{\Omega_{24}}{\alpha_{4}}\right)+\lambda_{3}\left(\frac{\Omega_{33}}{2 \alpha_{3}}-\frac{\Omega_{34}}{\alpha_{4}}\right)-\frac{\lambda_{2} \lambda_{3}}{\alpha_{4} \lambda_{4}} \Omega_{23} .
$$

The last equation describes the renormalization group running of the Barbero-Immirzi parameter within the on-shell renormalization group scheme. In this consideration we assumed 
that the angle between the four-dimensional currents $J^{\mu}$ and $W^{\mu}$ does not run with the renormalization group scale. This is a small price to pay for the possibility to consider renormalization group in the non-renormalizable theory such as Einstein-Cartan gravity with the Holst term.

The problem of exploring the asymptotic behavior of the effective charges $\lambda_{2,3,4}(t)$ and $\gamma(t)$ on the basis of eqs. (49), (50), (51) and (58) turns out to be very complicated, and unfortunately we were unable to solve it in a completely satisfactory way. Let us present only some part of consideration, which can be useful to show what is the origin of the difficulties.

The simplest assumption is that all four parameters $\lambda_{2,3,4}(t)$ and $\gamma(t)$ have moderate running and therefore one can work in the leading-log approximation. Then the eqs. (49) and (50) can be easily solved for a constant $\gamma$ and give

$$
\lambda_{2}(t)=\frac{\lambda_{20}}{1+b_{2}^{2} \lambda_{20} t}, \quad \lambda_{3}(t)=\frac{\lambda_{30}}{1-b_{3}^{2} \lambda_{30} t} .
$$

In this case equation (51) can be easily cast into the form

$$
\frac{d \lambda_{4}}{d t}=A(t) \lambda_{4}^{2}+B(t) \lambda_{4}+C(t)
$$

Mathematically, (60) is a Riccati equation, which can be solved if we first get some particular solution. In order to achieve this, we can make some simplifications. Consider an asymptotic regime, assuming $\left(\beta_{2} / \lambda_{2}^{0}\right) t \gg 1$ and $\left(\beta_{3} / \lambda_{3}^{0}\right) t \gg 1$, such that, approximately,

$$
\lambda_{2,3}(t)=\frac{l_{2,3}}{t}, \quad \text { where } \quad l_{2}=\frac{4(4 \pi)^{2}\left(1+\gamma^{2}\right)}{27 \gamma^{2}} \quad \text { and } \quad l_{3}=16 l_{2} .
$$

In this way eq. (60) becomes simpler,

$$
\frac{d \lambda_{4}}{d t}=A_{0} \lambda_{4}^{2}+\frac{B_{0}}{t} \lambda_{4}+\frac{C_{0}}{t^{2}}
$$

where

$$
A_{0}=-\frac{\Omega_{44}}{\alpha_{4}(4 \pi)^{2}}, \quad B_{0}=-\frac{\Omega_{24} l_{2}+\Omega_{34} l_{3}}{\alpha_{4}(4 \pi)^{2}}, \quad C_{0}=-\frac{\Omega_{23} l_{2} l_{3}}{\alpha_{4}(4 \pi)^{2}} .
$$

It is quite natural to look for a particular solution of eq. (62) in the form

$$
\lambda_{4}(t)=\frac{l_{4}}{t}, \quad \text { where } \quad l_{4}=-\frac{B_{0}+1}{2 A_{0}} \pm \frac{1}{2 A_{0}} \sqrt{\left(B_{0}+1\right)^{2}-4 C_{0} A_{0}} .
$$

In case of a real r.h.s. of the last expression, one can easily show that an arbitrary particular solution is asymptotically approaching (64). Unfortunately, a direct calculus shows that the root in eq. (64) has only solutions with non-zero imaginary part. This feature leaves very 
small chances to find a fixed point for the system of equations (49), (50), (51) and (58). According to (37), the parameter $\lambda_{4}$ can be complex only due to the complex parameter $\gamma$. This means that the ratio between real and imaginary parts of $\lambda_{4}$ and $\gamma$ should be identically equal. However, direct calculations show that this situation contradicts the equations (51) and (58). This means that the system of renormalization group equations for the effective parameters $\lambda_{2,3,4}(t)$ and $\gamma(t)$ has no fixed points.

The absence of the Holst term means the limit $\gamma \rightarrow \infty$ for the Barbero-Immirzi parameter. An inspection of the eqs. (49), (50) and (51) with $\Omega_{22}$ and $\Omega_{33}$ defined in (42) shows that in this limit there are usual UV fixed points, which can correspond to the asymptotic freedom in the parameters $\lambda_{2}(t), \lambda_{3}(t)$ under the right choice of initial conditions. Therefore, the role of the Holst term in the renormalization group is very strong. Our results show that the presence of finite $\gamma$ breaks down the simple form of the renormalization group flows and leads to much more complicated scale behavior which looks irregular, at least at the present stage of investigating the problem.

In this situation a natural question to ask is whether the limit $\gamma \rightarrow \infty$ for the BarberoImmirzi parameter is smooth. It is easy to see that in this limit we also have $\lambda_{4} \rightarrow \infty$. Therefore the smooth limit concerns the ratio between the two effective parameters, $p=$ $\lambda_{4} / \gamma$. The equation for this ratio can be easily obtained from eqs. (37), (54) and (55). After a very small calculus we arrive at the equation

$$
\frac{d p}{d t}=-\frac{p}{2(4 \pi)^{2}}\left[\frac{\Omega_{22}}{\alpha_{2}} \lambda_{2}(t)+\frac{\Omega_{33}}{\alpha_{3}} \lambda_{3}(t)\right], \quad p(0)=p_{0} .
$$

Using the asymptotic estimates for $\gamma \rightarrow \infty$,

$$
\Omega_{22} \propto 81, \quad \Omega_{33} \propto \frac{81}{256}, \quad \alpha_{2} \propto 12, \quad \alpha_{3} \propto-\frac{3}{4}
$$

we arrive at the solution of (65),

$$
\frac{p(t)}{p_{0}} \propto\left(1+b_{2}^{2} \lambda_{20} t\right)^{-1 / 2}\left(1-b_{3}^{2} \lambda_{30} t\right)^{-1 / 2} .
$$

The last formula shows that we can switch off the Barbero-Immirzi parameter smoothly and the ratio $p(t) \rightarrow 0$ asymptotically at $t \rightarrow \infty$ in the same way as the effective charges $\lambda_{2}(t)$ and $\lambda_{3}(t)$. This shows that our hypothesis of a non-running angle between two currents is correct in the regime of very small Holst term, at least. However, this confirmation concerns only this special limit.

Of course, the most interesting part is the running for a finite Barbero-Immirzi parameter, but in this case we could not achieve a reliable analytic estimate of the results. In this situation one can rely only on the numerical solution for the system of equations (49), (50), 
(51) and (58). The corresponding analysis has been done, however the output shows very strong dependence on the choice of initial conditions and after all, there is no convincing qualitative interpretation of the results. For this reason, we decided not to bother the reader with the technical details here. One could imagine that the situation may become different in a more complete case when we also take the running of $\lambda_{1}$ into account. The technically more cumbersome analysis of this case have been performed and we saw that there are no much changes. Qualitatively, the situation remains the same, that is there are no nontrivial fixed points in the presence of finite Barbero-Immirzi parameter.

\section{Conclusions}

We have considered the Einstein-Cartan theory with an additional Holst term, which plays an important role in loop quantum gravity $[6,1]$. In classical theory this term is well-known to identically vanish for zero torsion, it manifest itself only in the presence of fermion currents. Following [10], we used the irreducible components of torsion to write the Holst term in a simple form, where its parity-violating nature becomes clear.

In the main part of the paper we performed one-loop calculations in the Einstein-Cartan theory with the Holst term, cosmological constant and two external fermion currents, namely with vector and axial vector ones. As one should expect, the divergences do not repeat the form of the classical action. On the other hand, the divergences have strong gauge-fixing dependence. In pure quantum GR one can chose the gauge-fixing in such a way that the one-loop $S$-matrix is becoming finite [29], however this in not the case if the matter is present, including fermions. Indeed, one does not need to calculate explicitly gaugefixing dependence, it is sufficient to remember that, at the one-loop level, this dependence disappears on the classical mass-shell in a general gauge theory [31] (see also [14] for a recent review of the subject).

The real problem is how to extract the potentially relevant physical information from the gauge-dependent effective action. One of the simplest possibilities has been suggested by Fradkin and Tseytlin in [19], where the truncated, on-shell, version of renormalization group equations has been introduced. Within this scheme one can arrive at the gaugeinvariant form of running for the dimensionless combination of the cosmological and Newton constants. The on-shell renormalization group has been also used in the Einstein-Cartan theory with axial vector current [21], but the situation becomes much more complicated and interesting in the presence of the Holst term.

In is clear that the on-shell renormalization group equations have much more restricted theoretical background than the conventional renormalization group in renormalizable theories. However, even in the non-renormalizable theory such as Einstein-Cartan with the 
Holst term we were able to establish the renormalization group equations for all dimensionless effective charges, including cosmological constant, squares of both fermionic currents, their mixing and finally, for the Barbero-Immirzi parameter $\gamma$. Unfortunately, the equations which we have obtained are very complicated and do not enable us to apply standard treatments. In particular, we were unable to find non-trivial UV fixed points in the theory or establish, by means of numerical methods, some reliable form of the renormalization group trajectories for the dimensionless effective charges.

Finally, let us present a short discussion of the perspectives to extend our results. The set of equations which we have obtained here, can be seen as a low-energy approximation for the renormalization group in the theory with full UV completion, which is supposed to be renormalizable. In the present case such a complete theory should include higher derivatives in the metric sector [32] and kinetic terms for torsion (see the discussion in [27, 22]). Only quantum calculations in such a full theory coupled to fermions [33, 34, 27] can provide a completely reliable form of the renormalization group equations in the theory with BarberoImmirzi parameter. In practise, the derivation of such equations is possible but promise to be very involved, so we leave it for the possible future work. At the same time, certain technical tools which we developed here will be certainly necessary for such a calculation.

\section{Acknowledgements}

Authors are grateful to Guilherme de Berredo-Peixoto and Cleber Abrahão de Souza for useful discussions about the Holst term. We are especially grateful to Prof. Friedrich Hehl, who explained us a mistake in the Eq. (12) in the first version of the manuscript and also provided us by some relevant references. The work of the authors has been partially supported by CAPES, CNPq, FAPEMIG and ICTP (I.Sh.).

\section{References}

[1] L. Freidel, D. Minic and T. Takeuchi, Phys. Rev. D 72 (2005) 104002.

[2] G. de Berredo-Peixoto, L. Freidel, I.L. Shapiro and C.A. de Souza, JCAP 06 (2012) 017, arXiv: 1201.5423 .

[3] O. Castillo-Felisola, C. Corral, C. Villavicencio and A.R. Zerwekh, Phys. Rev. D 88 (2013) 124022, arXiv: 1310.4124.

[4] S. Holst, Phys. Rev. D 53 (1996) 5966.

[5] A. Ashtekar, Phys. Rev. D 36 (1987) 1587. 
[6] A. Perez and C. Rovelli, Phys. Rev. D 73 (2006) 044013, gr-qc/0505081.

[7] S. Alexandrov, Class. Quant. Grav. 25 (2008) 145012, arXiv: 0802.1221.

[8] J.F. Barbero, Phys. Rev. D 51 (1995) 5507.

[9] G. Immirzi, Class. Quant. Grav. 14 (1997) L177.

[10] S. Mercuri, Phys. Rev. D 73 (2006) 084016, gr-qc/0601013.

M. Kazmierczak, Phys. Rev. D 79 (2009) 064029, arXiv: 0812.1298.

[11] I.B. Khriplovich and A.S. Rudenko, JCAP 1211 (2012) 040, arXiv: 1210.7306.

[12] J.E. Daum and M. Reuter, Annals Phys. 334 (2013) 351, arXiv: 1301.5135.

[13] H. Gies, Lect. Notes Phys. 852 (2012) 287, hep-th/0611146.

[14] P.M. Lavrov and I.L. Shapiro, JHEP 06 (2013) 086, arXiv: 1212.2577.

[15] D.J. Toms, Nature 468 (2010) 56, arXiv: 1010.0793.

J.C.C. Felipe, L.C.T. Brito, M. Sampaio and M.C. Nemes, Phys. Lett. B 700 (2011) 86, arXiv: 1103.5824 .

[16] D. Benedetti and S. Speziale, JHEP 06 (2011) 107, arXiv: 1104.4028. J. Phys. Conf. Ser. 360 (2012) 012011, arXiv: 1111.0884. Proceedings of "Loops 11: International Conference on Quantum Gravity", 23-28 May - 2011. Madrid, Spain.

[17] A.O. Barvinsky and G.A. Vilkovisky, Nucl. Phys. B 191 (1981) 237.

[18] G. t' Hooft and M.J.G. Veltman, Ann. Poincare Phys. Theor. A 20 (1974) 69.

[19] E.S. Fradkin and A.A. Tseytlin, Nucl. Phys. B 201 (1982) 469.

[20] E.S. Fradkin and A.A. Tseytlin, Nucl. Phys. B 234 (1984) 472.

[21] I.L. Buchbinder and I.L. Shapiro, Sov. J. Phys. 31 (1988) 40.

[22] I.L. Shapiro, Phys. Repts. 357 (2002) 113.

[23] F.W. Hehl, P. von der Heyde, G.D. Kerlick, and J.M. Nester, Rev. Mod. Phys. 48 (1976) 393.

[24] V. De Sabbata and M. Gasperini, Introduction To Gravity, (World Scientific, Singapore $-1985)$.

[25] R.T. Hammond, Rept. Prog. Phys. 65 (2002) 599. 
[26] I.L. Buchbinder and I.L. Shapiro, Phys. Lett. B 151 (1985) 263.

[27] I.L. Buchbinder, S.D. Odintsov and I.L. Shapiro, Effective Action in Quantum Gravity, (IOP Publishing, Bristol - 1992).

[28] P. Baekler, F.W. Hehl and J.M. Nester, Phys. Rev. D 83 (2011) 024001, arXiv: 1009.5112.

M. Blagojević and F.W. Hehl (Eds.), Gauge Theories of Gravitation, a Reader with Commentaries, (World Scientific, Singapore - 2013).

[29] R.E. Kallosh, O.V. Tarasov and I.V. Tyutin, Nucl. Phys. B 137 (1978) 145.

[30] I. L. Shapiro and A. G. Jacksenaev, Phys. Lett. B 324 (1994) 284.

[31] B.L. Voronov, P.M. Lavrov and I.V. Tyutin, Yad. Fiz. 36498 (1982); Sov. J. Nucl. Phys. 36292 (1982).

[32] K.S. Stelle, Phys. Rev. D 16 (1977) 953.

[33] I.L. Buchbinder, O.K. Kalashnikov, I.L. Shapiro, V.B. Vologodsky and Yu.Yu. Wolfengaut, Phys. Lett. B 216 (1989) 127.

[34] I.L. Shapiro, Class. Quant. Grav. 6 (1989) 1197. 